\title{
Arthroscopic Excision of Solitary Intra-articular Osteochondroma of the Knee
}

\author{
Jong In Kim, $\mathrm{MD}^{1}$, Jae Ho Kwon, $\mathrm{MD}^{1}$, Yong Jee Park, $\mathrm{MD}^{2}$, Vivian R. D’Almeida, $\mathrm{MS}^{3}$, Shaishav M. Soni, MS , \\ and Kyung Wook Nha, $\mathrm{MD}^{1}$ \\ ${ }^{1}$ Department of Orthopedic Surgery, Inje University Ilsan Paik Hospital, Goyang; ${ }^{2}$ Department of Orthopedic Surgery, Bang Hwa Hospital, Seoul, Korea; ${ }^{3}$ Department \\ of Orthopedic Surgery, Fr. Muller Medical College Hospital, Mangalore; ${ }^{4}$ Department of Orthopedic Surgery, Siddhi Vinayak Hospital, Ahmedabad, India
}

Osteochondroma is the most common benign tumor of the growing bone commonly involving the knee joint region. It often involves the metaphysis of the long bone, occurring extra-articularly. In spite of this, solitary intra-articular osteochondroma has rarely been reported in the literature. A 41-year-old man presented with diffuse pain and discomfort of the left knee for over 2 months. Clinical examination revealed a bony prominence involving the supero-lateral aspect of the left distal femur. Diagnostic evaluation involved radiography, magnetic resonance imaging, and a diagnostic arthroscopy, which showed features of an intra-articular osteochondroma in the left distal femur. Arthroscopic excision of the solitary intra-articular osteochondroma resulted in a complete relief of symptoms and return to full competitive activities. No recurrence of symptoms occurred during the one year of follow-up. Solitary intra-articular osteochondroma of the knee is an unusual case, which can be successfully managed with arthroscopy.

Keywords: Osteochondroma, Intra-articular, Arthroscopy, Knee

Osteochondroma is the most common benign bone tumor and is characterised by a cartilaginous cap occurring mainly in the growing age with the knee ${ }^{1}$. They likely are developmental malformations rather than true neoplasms and are thought to originate within the periosteum as small cartilaginous nodules. The tumor is usually located extra-articularly in the proximal femur and proximal humerus, and is asymptomatic. Intraarticular osteochondromas of the hip and the ankle joint have been reported, which are rare ${ }^{2)}$. In cases involving the knee joint, they are para-articular, extra-skeletal osteochondromas ${ }^{3)}$.

We report a patient who presented with pain and discomfort

Received October 22, 2012; Revised November 28, 2012;

Accepted December 21, 2012

Correspondence to: Kyung Wook Nha, MD

Department of Orthopedic Surgery, Inje University Ilsan Paik Hospital, Inje University College of Medicine, 170 Juhwa-ro, Ilsanseo-gu, Goyang 411-706, Korea

Tel: +82-31-910-7968, Fax: +82-31-910-7967

E-mail:kwnhamj@hotmail.com

This is an Open Access article distributed under the terms of the Creative Commons Attribution Non-Commercial License (http://creativecommons.org/licenses/by-nc/3.0/) which permits unrestricted non-commercial use, distribution, and reproduction in any medium, provided the original work is properly cited. due to solitary intra-articular osteochondroma with connecting femur in the knee joint, which was relieved by arthroscopic excision.

\section{Case Report}

A 41-year-old man presented with diffuse pain and discomfort in the left knee for two months. He did not report any trauma or medical problem. Physical examination revealed a palpable bony hard mass of approximately $2 \times 2 \mathrm{~cm}$ in size over the superolateral aspect of the left distal femur. The skin was normal. There was no restriction in the range of movements of the knee joint, but pain was reported with over 90 degree flexion of the knee. The Lysholm score was 80, and the International Knee Documentation Committee (IKDC) score was 79 points.

Simple radiography showed a bony mass arising from the supero-lateral aspect of the left distal femur without stalk (Fig. 1). Magnetic resonance imaging (MRI) showed a prominence with the same intensity as a bone, arising from distal femur within the joint capsule. Cortical bone was communicating into the medullary canal of the femur. A characteristic cartilaginous cap was also seen (Fig. 2).

Under general anesthesia, arthroscopic standard portals were 
made, and the superolateral view revealed an intra-articular cartilaginous cap. There was minimal synovitis of the joint. The cartilage cap was connected to the femur (Fig. 3). Ligaments,

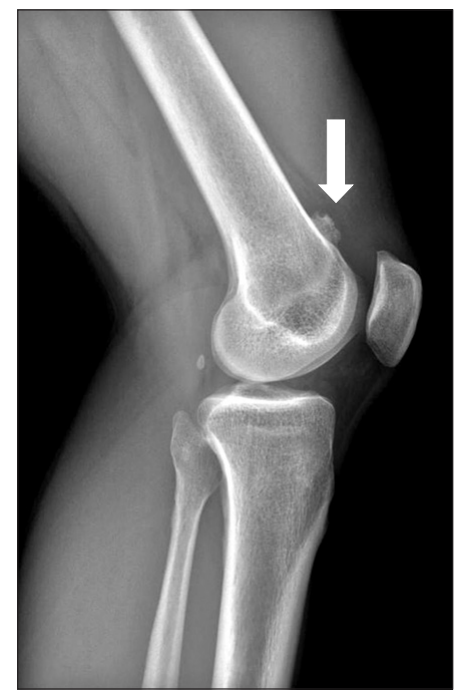

Fig. 1. Simple radiography shows a benign tumor involving the distal femur with a stalk (arrow). cartilage, and menisci were intact, and patellar tracking was normal in a full range of knee motion. The tumor was then excised using a small osteotomy at the supermedial portal.

The histopathologic report showed that the bony outgrowth was mainly a cancellous bone with a cartilaginous cap, which is a feature consistent with osteochondroma (Fig. 4). At one year follow-up, the patient was asymptomatic and involved in full activities. In addition, the Lysholm score and IKDC score were both 100. Radiologic follow-up revealed no recurrence (Fig. 5).

\section{Discussion}

Intra-articular osteochondroma of the knee is a rare tumor. Arthroscopic excision of a symptomatic osteochondroma may be less painful and results in a faster recovery than the open incision approach.

Generally, osteochondromas occur around the growth plate of long bones in a skeletally immature person and move towards the diaphysis with the connected bone. Therefore, it is rare that osteochondroma is located within the articular compartment of a joint in an adult ${ }^{3)}$. While intra-articular osteochondromas
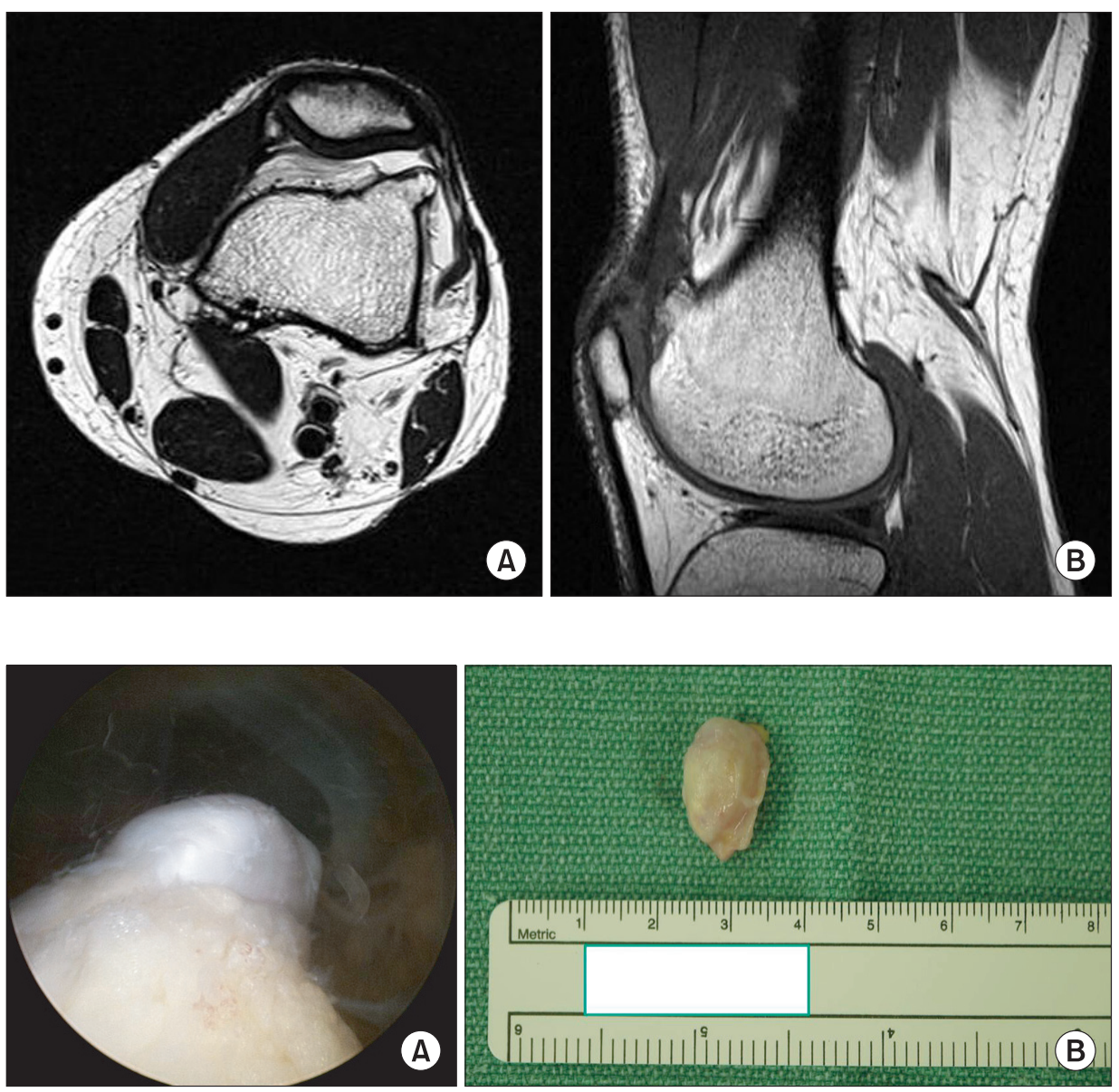

Fig. 2. Magnetic resonance images (A, axial image; $B$, sagittal image) show an intra-articular osteochondroma with the cancellous bone communicating with the medullary canal.

Fig. 3. (A) Arthroscopic superolateral view shows the intra-articular cartilagenous cap of the knee joint. (B) The gross shape of lesion was seen. 


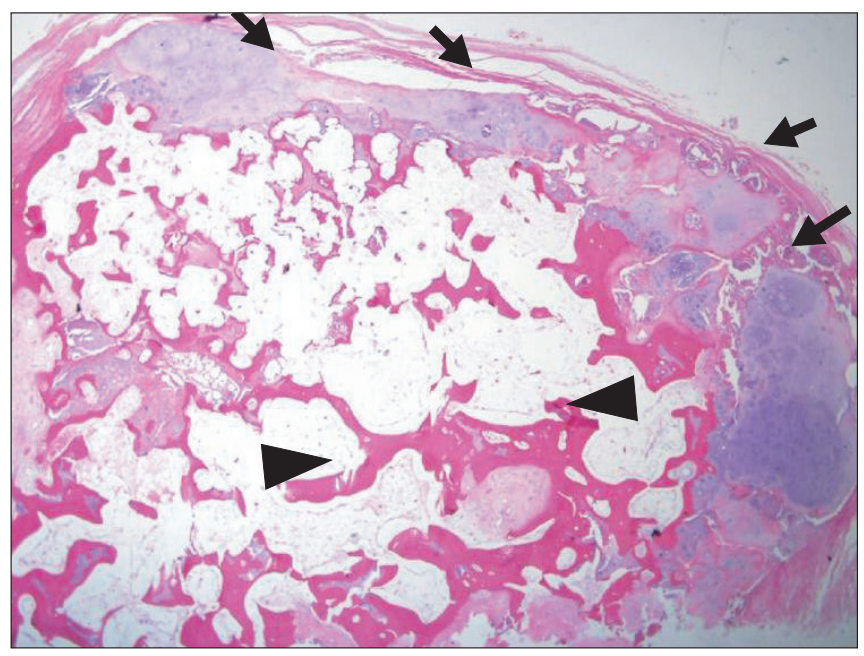

Fig. 4. Histopathology shows the cartilage cap appears pale blue (arrows), with underlying bone appearing pink (arrowhead) (H\&E, $\times 40)$.

of the hip and ankle joint have been reported, these cases are rare $^{2)}$. Intra-articular osteochondroma in the knee joint has been reported only in two cases ${ }^{4,5)}$. Schmoyer and Ciullo ${ }^{4)}$ reported a case which was not intra-articular osteochondroma. They used an image intensifier to confirm the location, dissected the bony mass, and performed arthroscopic resection. While Takahashi et al. ${ }^{5)}$ resected the intra-articular osteochondroma by arthroscopy, their case involved multiple osteochondromas. In this case, simple radiography and whole body bone scan were normal in the other commonly involved sites, such as shoulder, hip, and ankle. To our knowledge, solitary intra-articular osteochondroma in the knee joint is a very rare case. In addition, the arthroscopic excision of osteochondroma of knee joint has been reported rarely.

While the extra-articular tumors are usually symptomless, intraarticular tumors cause pain and discomfort with restrictions in the range of movements. Differential diagnoses include synovial chondromatosis, low-grade chondrosarcoma, and osteosarcoma. MRI imaging helps to differentiate these lesions ${ }^{6}$.

The diagnosis of the tumors are made by a combination of clinical signs, plain radiography, and MRI. MRI usually shows a well-circumscribed lesion with signal intensity similar to that of a bone with a cartilaginous cap. MRI of the this patient demonstrates diagnostic marrow and cortex continuity of a sessile osteochondroma (Fig. 2). The size of the cap is very important to rule out malignant transformation, as a cap larger than 1 $\mathrm{cm}$ suggests malignancy. Microscopic examination shows a cap of mature hyaline cartilaginous tissue covered by a fibrous membrane. The centre of the lesion consisted of mature bone trabeculae located beneath the cartilaginous cap containing

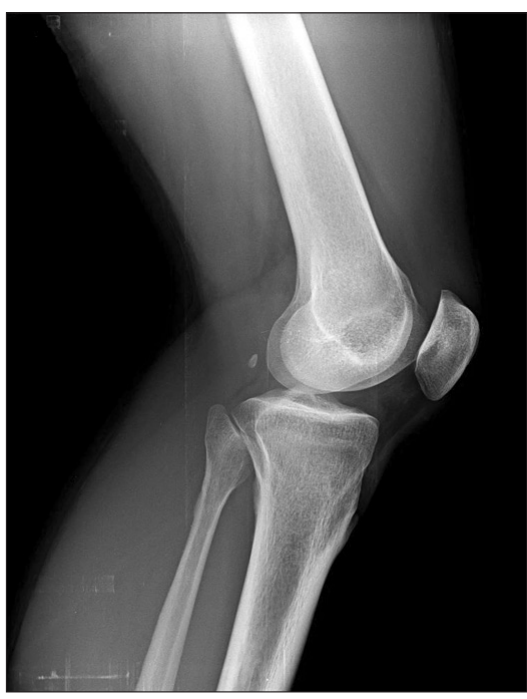

Fig. 5. After 1 year, simple radiography shows no recurrence.

bone marrow and amorphous calcified debris. To further rule out malignancy, cellular atypia and mitotic activity need to be evaluated $^{7}$. This case also showed matured bone trabeculae with a cartilaginous cap (Fig. 4).

Surgical excision is the treatment of choice for osteochondromas $^{3)}$. This can be performed via an open technique or arthroscopically. Recurrences mostly have not been reported in the literature ${ }^{1)}$.

In conclusion, solitary intra-articular osteochondroma of the knee is an unusual case, which can be successfully managed with arthroscopy.

\section{Conflict of Interest}

No potential conflict of interest relevant to this article was reported.

\section{Acknowledgments}

This work was supported by 2013 grant from Inje University.

\section{References}

1. Reith JD, Bauer TW, Joyce MJ. Paraarticular osteochondroma of the knee: report of 2 cases and review of the literature. Clin Orthop Relat Res. 1997;(334):225-32.

2. Siebenrock KA, Ganz R. Osteochondroma of the femoral neck. Clin Orthop Relat Res. 2002;(394):211-8.

3. Rizzello G, Franceschi F, Meloni MC, Cristi E, Barnaba SA, Rabitti C, Denaro V. Para-articular osteochondroma of the 
knee. Arthroscopy. 2007;23:910.e1-4.

4. Schmoyer S, Ciullo JV. Arthroscopic resection of an osteochondroma of the knee. Arthroscopy. 2001;17:765-7.

5. Takahashi M, Nishihara A, Ohishi T, Shiga K, Yamamoto K, Nagano A. Arthroscopic resection of an intra-articular osteochondroma of the knee in the patient with multiple osteochondromatosis. Arthroscopy. 2004;20 Suppl 2:28-31.

6. Giudici M, Moser R, Kransdorf M. Cartilaginous bone tumors. Radiol Clin North Am. 1993;31:237-59.

7. Garrison RC, Unni KK, McLeod RA, Pritchard DJ, Dahlin DC. Chondrosarcoma arising in osteochondroma. Cancer. 1982;49:1890-7. 